\title{
Semiarcs with long secants
}

\author{
Bence Csajbók* \\ Department of Mathematics, Informatics and Economics \\ University of Basilicata \\ Potenza, Italy \\ bence.csajbok@unibas.it
}

Submitted: Oct 1, 2013; Accepted: Mar 3, 2014; Published: Mar 17, 2014

Mathematics Subject Classifications: 51E20, 51E21

\begin{abstract}
In a projective plane $\Pi_{q}$ of order $q$, a non-empty point set $\mathcal{S}_{t}$ is a $t$-semiarc if the number of tangent lines to $\mathcal{S}_{t}$ at each of its points is $t$. If $\mathcal{S}_{t}$ is a $t$-semiarc in $\Pi_{q}, t<q$, then each line intersects $\mathcal{S}_{t}$ in at most $q+1-t$ points. Dover proved that semiovals (semiarcs with $t=1$ ) containing $q$ collinear points exist in $\Pi_{q}$ only if $q \leqslant 3$. We show that if $t>1$, then $t$-semiarcs with $q+1-t$ collinear points exist only if $t \geqslant \sqrt{q-1}$. In $\operatorname{PG}(2, q)$ we prove the lower bound $t \geqslant(q-1) / 2$, with equality only if $\mathcal{S}_{t}$ is a blocking set of Rédei type of size $3(q+1) / 2$.

We call the symmetric difference of two lines, with $t$ further points removed from

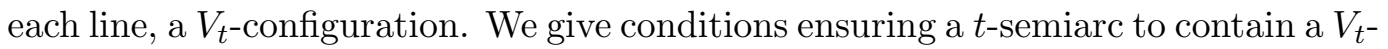
configuration and give the complete characterization of such $t$-semiarcs in $\mathrm{PG}(2, q)$.
\end{abstract}

Keywords: collineation group; blocking set; semioval

\section{Introduction}

Semiarcs are natural generalizations of arcs. Let $\Pi_{q}$ be a projective plane of order $q$. A non-empty point set $\mathcal{S}_{t} \subset \Pi_{q}$ is called a $t$-semiarc if for every point $P \in \mathcal{S}_{t}$ there exist exactly $t$ lines $\ell_{1}, \ell_{2}, \ldots, \ell_{t}$ such that $\mathcal{S}_{t} \cap \ell_{i}=\{P\}$ for $i=1,2, \ldots, t$. These lines are called the tangents to $\mathcal{S}_{t}$ at $P$. If a line $\ell$ meets $\mathcal{S}_{t}$ in $k>1$ points, then $\ell$ is called a $k$-secant of $\mathcal{S}_{t}$. The classical examples of semiarcs are the semiovals (semiarcs with $t=1$ ) and point sets of type $(0,1, n)$ (i.e. point sets meeting each line in either 0 , or 1 , or $n$ points, in this case $t=q+1-(s-1) /(n-1)$, where $s$ denotes the size of the point set). Arcs, unitals, and subplanes are semiarcs of the latter type. For more examples, see [1], [5] and [10].

\footnotetext{
*Author was supported by the Hungarian National Foundation for Scientific Research, Grant No. K 81310 .
} 
Because of the huge diversity of the geometry of semiarcs, their complete classification is hopeless. In [7] Dover investigated semiovals with a $q$-secant and semiovals with more than one $(q-1)$-secant. The aim of this paper is to generalize these results and characterize $t$-semiarcs with long secants.

Many of the known $t$-semiarcs contain the symmetric difference of two lines, with $t$ further points removed from each line. We will call this set of $2(q-t)$ points a $V_{t}$-configuration. Recently in [5] it was proved that in $\mathrm{PG}(2, q)$ small semiarcs with a long secant necessarily contain a $V_{t}$-configuration or can be obtained from a blocking set of Rédei type. Here we give another condition ensuring a $t$-semiarc to contain a $V_{t}$-configuration and we give the complete characterization of such $t$-semiarcs in $\operatorname{PG}(2, q)$. To do this we use the classification of perspective point sets in $\mathrm{PG}(2, q)$. This is a result due to Korchmáros and Mazzoca [11] and it is related to Dickson's classification of the subgroups of the affine group on the line $\mathrm{AG}(1, q)$.

Using a result of Weiner and Szönyi, that was conjectured by Metsch, we prove that $t$-semiarcs in $\mathrm{PG}(2, q)$ with $q+1-t$ collinear points exist if and only if $t \geqslant(q-1) / 2$. The case of equality is strongly related to blocking sets of Rédei type, we also discuss these connections.

If $t=q+1, q$ or $q-1$, then $\mathcal{S}_{t}$ is single point, a subset of a line or three non-collinear points respectively. To avoid trivial cases, we may assume for the rest of this paper that $t<q-1$.

\section{Semiarcs with one long secant}

If $\mathcal{S}_{t}$ is a $t$-semiarc in $\Pi_{q}, t<q$, then each line intersects $\mathcal{S}_{t}$ in at most $q+1-t$ points. In this section we study $t$-semiarcs containing $q+1-t$ collinear points. The following lemma gives an upper bound for the size of such $t$-semiarcs.

Lemma 1. If $\mathcal{S}_{t}$ is a t-semiarc in $\Pi_{q}$ and $\ell$ is a $(q+1-t)$-secant of $\mathcal{S}_{t}$, then $\left|\mathcal{S}_{t} \backslash \ell\right| \leqslant q$.

Proof. Let $U=\mathcal{S}_{t} \backslash \ell$ and let $D=\ell \backslash \mathcal{S}_{t}$. Through each point of $U$ there pass exactly $t$ tangents to $\mathcal{S}_{t}$ and each of them intersects $\ell$ in $D$. This implies $t|U| \leqslant q|D|$. Since $|D|=t$, we have $|U| \leqslant q$.

In [7] Dover proved that semiovals with a $q$-secant exist in $\Pi_{q}$ if and only if $q \leqslant 3$. Our first theorem generalizes this result and shows that if $\mathcal{S}_{t}$ has a $(q+1-t)$-secant, then $t$ cannot be arbitrary. For related ideas of the proof, see the survey paper by Blokhuis et. al. [3], Theorem 3.2.

Theorem 2. If $\mathcal{S}_{t}$ is a t-semiarc in $\Pi_{q}$ with a $(q+1-t)$-secant, then $t=1$ and $q \leqslant 3$ or $t \geqslant \sqrt{q-1}$.

Proof. Let $\ell$ be a line that satisfies $\left|\mathcal{S}_{t} \cap \ell\right|=q+1-t$ and let $U=\mathcal{S}_{t} \backslash \ell$. The size of $U$ has to be at least $q-t$, otherwise the points of $\ell \cap \mathcal{S}_{t}$ would have more than $t$ tangents. This and Lemma 1 together yield:

$$
q-t \leqslant|U| \leqslant q
$$


Let $q-t+k$ be the size of $U$, where $0 \leqslant k \leqslant t$. Let $\delta$ be the number of lines that do not meet $U$ and denote by $L_{1}, L_{2}, \ldots, L_{q^{2}+q+1-\delta}$ the lines that meet $U$. For these lines let $e_{i}=\left|L_{i} \cap U\right|$. The standard double counting argument gives:

$$
\begin{aligned}
\sum_{i=1}^{q^{2}+q+1-\delta} e_{i} & =(q-t+k)(q+1), \\
\sum_{i=1}^{q^{2}+q+1-\delta} e_{i}\left(e_{i}-1\right) & =(q-t+k)(q-t+k-1) .
\end{aligned}
$$

If a line $\ell^{\prime}$ intersects $U$ in more than one point, then $Q:=\ell^{\prime} \cap \ell$ is in $\mathcal{S}_{t}$, otherwise the points of $\ell^{\prime} \cap U$ would have at most $t-1$ tangents. The point $Q \in \mathcal{S}_{t}$ has at least $q-1-\left(q-t+k-\left|\ell^{\prime} \cap U\right|\right)=t-1-k+\left|\ell^{\prime} \cap U\right|$ tangents, hence $\left|\ell^{\prime} \cap U\right| \leqslant k+1$. This implies $e_{i} \leqslant k+1$, for $i=1,2, \ldots, q^{2}+q+1-\delta$, thus the following holds:

$$
\sum_{i=1}^{q^{2}+q+1-\delta} e_{i}\left(e_{i}-1\right) \leqslant(k+1) \sum_{i=1}^{q^{2}+q+1-\delta}\left(e_{i}-1\right)=(k+1)\left((q-t+k)(q+1)-\left(q^{2}+q+1-\delta\right)\right) .
$$

The line $\ell$ does not meet $U$ and the other lines that do not meet $U$ fall into two classes: there are $(q+1-t) t$ of them passing through $\ell \cap \mathcal{S}_{t}$ (the tangents to $\mathcal{S}_{t}$ through the points of $\left.\ell \cap \mathcal{S}_{t}\right)$ and there are $t q-(q-t+k) t$ of them passing through $\ell \backslash \mathcal{S}_{t}$ (the lines intersecting $\ell \backslash \mathcal{S}_{t}$ minus the tangents to $\mathcal{S}_{t}$ through the points of $\left.U\right)$. This implies $\delta=t(q+1-k)+1$, hence we can write (4) as:

$$
(q-t+k)(q-t+k-1) \leqslant(k+1)\left((q-t+k)(q+1)-\left(q^{2}+q\right)+t(q+1-k)\right) .
$$

Rearranging this inequality we obtain:

$$
q^{2}-q\left(2 t+1-k+k^{2}\right)+k^{2} t-k t-2 k+t^{2}+t \leqslant 0 .
$$

The discriminant of the left-hand side polynomial is $k^{4}-2 k^{3}+3 k^{2}+6 k+1$. If $k=0,1,2$, then we get $q \leqslant t+1, t+2, t+4$ respectively. Otherwise, we have $k^{4}-2 k^{3}+3 k^{2}+6 k+1<$ $\left(k^{2}-k+3\right)^{2}$, which yields $q \leqslant t+k^{2}-k+1$. The maximum value of $k$ is $t$, therefor $q \leqslant t^{2}+1$ follows for $k \geqslant 3$. If $t=1$, then $k \leqslant 1$, hence $q \leqslant t+2=3$. If $t=2$, then $k \leqslant 2$, hence $q \leqslant t+4=6$. Since there is no projective plane of order 6 , in this case we get $q \leqslant 5$. If $t \geqslant 3$ and $k<3$, then $q \leqslant t+4<t^{2}+1$ and this completes the proof.

Before we go further we need some definitions about blocking sets. A blocking set of a projective plane is a point set $\mathcal{B}$ that intersects every line in the plane. A blocking set is minimal if it does not contain a smaller blocking set and it is non-trivial if it does not contain a line. If $\mathcal{B}$ is a non-trivial blocking set, then we have $|\ell \cap \mathcal{B}| \leqslant|\mathcal{B}|-q$ for every line $\ell$. If there is a line $\ell$ such that $|\ell \cap \mathcal{B}|=|\mathcal{B}|-q$, then $\mathcal{B}$ is a blocking set of Rédei type and the line $\ell$ is a Rédei line of $\mathcal{B}$.

In $\mathrm{PG}(2, q)$ we can improve the bound in Theorem 2. To do this we use the following result, conjectured by Metsch [13] and proven by Weiner and Szőnyi in [15, 16]. 
Theorem $3([15,16])$. Let $U$ be a point set in $\mathrm{PG}(2, q), P$ a point not in $U$ and assume that there pass exactly $r$ lines through $P$ meeting $U$. Then the total number of lines meeting $U$ is at most $1+r q+(|U|-r)(q+1-r)$.

Theorem 4. Let $\mathcal{S}_{t}$ be a t-semiarc in $\mathrm{PG}(2, q)$. If $\mathcal{S}_{t}$ has a $(q+1-t)$-secant, then $t \geqslant(q-1) / 2$. In the case of equality, $\mathcal{S}_{t}$ is a blocking set of Rédei type and its $(q+1-t)$ secants are Rédei lines.

Proof. Let $\ell$ be a $(q+1-t)$-secant of $\mathcal{S}_{t}$ and let $U=\mathcal{S}_{t} \backslash \ell$. From Lemma 1 , we have:

$$
|U| \leqslant q .
$$

The following statements are easy to check:

- the lines intersecting $U$ in more than one point intersect $\ell$ in $\ell \cap \mathcal{S}_{t}$,

- through each point of $\ell \cap \mathcal{S}_{t}$ there pass exactly $r=q-t$ lines meeting $U$,

- the total number of lines meeting $U$ is $\delta=|U| t+(q+1-t)(q-t)$.

Applying Theorem 3 for the point set $U$ and for a point $P \in \ell \cap \mathcal{S}_{t}$, we obtain:

$$
\delta=|U| t+(q+1-t)(q-t) \leqslant 1+(q-t) q+(|U|-q+t)(t+1) .
$$

After rearranging, we get:

$$
2 q-2 t-1 \leqslant|U| .
$$

Equations (6) and (8) together imply $t \geqslant(q-1) / 2$. If $t=(q-1) / 2$, then $|U|=q$ and there are $\delta=\left(3 q^{2}+2 q+3\right) / 4$ lines meeting $U$ and $(q+1-t) t=\left(q^{2}+2 q-3\right) / 4$ lines meeting $\ell \cap \mathcal{S}_{t}$ but not $U$. Together with the line $\ell$ we get the total number of lines in $\operatorname{PG}(2, q)$, thus $\mathcal{S}_{t}$ is a blocking set of Rédei type and $\ell$ is a Rédei line of $\mathcal{S}_{t}$.

The following result by Blokhuis yields another connection between blocking sets and semiarcs.

Theorem 5 ([2]). If $\mathcal{B}$ is a minimal non-trivial blocking set in $\mathrm{PG}(2, p), p>2$ prime, then $|\mathcal{B}| \geqslant 3(p+1) / 2$. In the case of equality there pass exactly $(p-1) / 2$ tangent lines through each point of $\mathcal{B}$.

Example 6 ([9], Lemma 13.6). Denote by $C$ the set of non-zero squares in $\operatorname{GF}(q), q$ odd, and let $\mathcal{S}_{t}=\{(c, 0,1),(0,-c, 1),(c, 1,0): c \in C\} \cup\{(1,0,0),(0,1,0),(0,0,1)\}$. This point set is called projective triangle and it is a $t$-semiarc with three $(q+1-t)$-secants, where $t=(q-1) / 2$. This example shows the sharpness of Theorems 4 and 5 .

In $\mathrm{PG}(2, q), q$ prime, Lovász and Schrijver proved that blocking sets of Rédei type of size $3(q+1) / 2$ are projectively equivalent to the projective triangle, see [12]. Gács, Lovász, and Szőnyi proved the same if $q$ is a square of a prime, see [8]. These results and Theorem 4 together yield the following:

Corollary $7([8,12])$. Let $\mathcal{S}_{t}$ be a t-semiarc in $\mathrm{PG}(2, q)$ with a $(q+1-t)$-secant. If $t=(q-1) / 2$ and $q=p$ or $q=p^{2}, p$ prime, then $\mathcal{S}_{t}$ is projectively equivalent to the projective triangle. 


\section{$3 \quad$ Semiarcs with two long secants}

Throughout the paper, if $A$ and $B$ are two point sets in $\Pi_{q}$, then $A \triangle B$ denotes their symmetric difference, that is $(A \backslash B) \cup(B \backslash A)$.

Definition 8. A $V_{t}$-configuration is the symmetric difference of two lines, with $t$ further points removed from both lines. Semiarcs containing a $V_{t}$-configuration fall into two types. Let $\mathcal{S}_{t}$ be a $t$-semiarc and suppose that there are two lines, $\ell_{1}$ and $\ell_{2}$, such that $\left(\ell_{1} \triangle \ell_{2}\right) \cap \mathcal{S}_{t}$ is a $V_{t}$-configuration, then:

- $\mathcal{S}_{t}$ is of $V_{t}^{\circ}$ type if $\ell_{1} \cap \ell_{2} \notin \mathcal{S}_{t}$,

- $\mathcal{S}_{t}$ is of $V_{t}^{\bullet}$ type if $\ell_{1} \cap \ell_{2} \in \mathcal{S}_{t}$.

For semiovals, Dover proved the following characterization:

Theorem 9 ([7], Lemma 4.1, Theorem 4.2). Let $\mathcal{S}_{1}$ be a semioval in $\Pi_{q}$. If $\mathcal{S}_{1}$ is of $V_{1}^{\circ}$ type, then it is contained in a vertexless triangle. If $q>5$ and $\mathcal{S}_{1}$ has at least two $(q-1)$-secants, then $\mathcal{S}_{1}$ is of $V_{1}^{\circ}$ type.

As the above result suggests, the characterization of $t$-semiarcs with two $(q-t)$ secants works nicely only for semiarcs of $V_{t}^{\circ}$ type. In Proposition 11 we generalize the last statement of the above result, but the characterization of $V_{t}^{\circ}$ type semiarcs seems to be hopeless in general. In Proposition 12 we consider the case when $t=2$, but for larger values of $t$ we deal only with the Desarguesian case, see Section 4.

Lemma 10. Let $\mathcal{S}_{t}$ be a $t$-semiarc in $\Pi_{q}, t<q$, and suppose that there exist two lines, $\ell_{1}$ and $\ell_{2}$, with their common point in $\mathcal{S}_{t}$ such that $\left|\ell_{1} \backslash\left(\mathcal{S}_{t} \cup \ell_{2}\right)\right|=n$ and $\left|\ell_{2} \backslash\left(\mathcal{S}_{t} \cup \ell_{1}\right)\right|=m$. Then $q \leqslant t+1+n m / t$ and $\left|\mathcal{S}_{t} \backslash\left(\ell_{1} \cup \ell_{2}\right)\right|=q-1-t$ in the case of equality.

Proof. Since $\mathcal{S}_{t}$ is not contained in a line, we have $n, m \geqslant t$. If one of $n$ or $m$ is equal to $q$, then $q<q+t+1 \leqslant t+1+n m / t$ and the assertion follows. Thus we can assume that $\ell_{1}$ and $\ell_{2}$ are not tangents to $\mathcal{S}_{t}$. Let $X=\mathcal{S}_{t} \backslash\left(\ell_{1} \cup \ell_{2}\right)$. Through the point $\ell_{1} \cap \ell_{2}$ there pass exactly $t$ tangents to $\mathcal{S}_{t}$, hence $q-1-t \leqslant|X|$. Through the points of $X$ there pass $|X| t$ tangents to $\mathcal{S}_{t}$, each of them intersects $\ell_{1}$ and $\ell_{2}$ off $\mathcal{S}_{t}$, hence $|X| t \leqslant n m$. These two inequalities imply $q \leqslant t+1+n m / t$ and $|X|=q-1-t$ in the case of equality.

Proposition 11. Let $\mathcal{S}_{t}$ be a t-semiarc in $\Pi_{q}$. If $\mathcal{S}_{t}$ has at least two $(q-t)$-secants and $q>2 t+3$, then $\mathcal{S}_{t}$ is of $V_{t}^{\circ}$ type. If $\mathcal{S}_{t}$ has at least two $(q-t+1)$-secants, then $\mathcal{S}_{t}$ is of $V_{t}^{\bullet}$ type.

Proof. If $\mathcal{S}_{t}$ has at least two $(q-t)$-secants with their common point in $\mathcal{S}_{t}$, then Lemma 10 implies $q \leqslant t+1+(t+1)^{2} / t=2 t+3+1 / t$. If $q>2 t+3$, then this is only possible when $t=1$ and $q=6$, but there is no projective plane of order 6 . Hence the common point of the $(q-t)$-secants is not contained in $\mathcal{S}_{t}$, which means that $\mathcal{S}_{t}$ is of $V_{t}^{\circ}$ type. The proof of the second statement is straightforward.

Proposition 12. Let $\mathcal{S}_{t}$ be a t-semiarc of $V_{t}^{\circ}$ type in $\Pi_{q}$. Then the following hold. 
(a) $\left|\mathcal{S}_{t}\right| \neq 2 q-2 t+1$.

(b) If $t=2$, then $\mathcal{S}_{t}$ is a $V_{2}$-configuration or $\left|\mathcal{S}_{t}\right|=2 q-2$ and $\mathcal{S}_{t}=\left(\ell_{1} \cup \ell_{2}\right) \triangle \Pi_{2}$, where $\ell_{1}$ and $\ell_{2}$ are two lines in $\Pi_{2}$, that is a Fano subplane contained in $\Pi_{q}$.

(c) If $t>1$, then $\left|\mathcal{S}_{t}\right| \leqslant 2 q-t$.

Proof. Let $\mathcal{S}_{t}$ be a $t$-semiarc of $V_{t}^{\circ}$ type and let $\ell_{1}$ and $\ell_{2}$ be two $(q-t)$-secants of $\mathcal{S}_{t}$ such that $P:=\ell_{1} \cap \ell_{2}$ is not contained in $\mathcal{S}_{t}$. Denote the points of $\ell_{1} \backslash\left(\mathcal{S}_{t} \cup P\right)$ by $A_{1}, \ldots, A_{t}$, the points of $\ell_{2} \backslash\left(\mathcal{S}_{t} \cup P\right)$ by $B_{1}, \ldots, B_{t}$. Let $X=\mathcal{S}_{t} \backslash\left(\ell_{1} \cup \ell_{2}\right)$ and define the line set $\mathcal{L}:=\left\{A_{i} B_{j}: 1 \leqslant i, j \leqslant t\right\}$ of size $t^{2}$. Through each point $Q \in X$ there pass exactly $t$ lines of $\mathcal{L}$, otherwise there would be an index $i \in\{1,2, \ldots, t\}$ for which the line $Q A_{i}$ meets $\ell_{2}$ in $\mathcal{S}_{t}$. But then there would be at most $t-1$ tangents to $\mathcal{S}_{t}$ through the point $Q A_{i} \cap \ell_{2}$, a contradiction.

Suppose, contrary to our claim, that $X$ consists of a unique point denoted by $Q$. Then $Q$ would have $t+1$ tangents: the $t$ lines of $\mathcal{L}$ that pass through $Q$ and the line $P Q$.

If $t=2$, then exactly two of the points of $\Pi_{q} \backslash\left(\ell_{1} \cup \ell_{2}\right)$ are contained in two lines of $\mathcal{L}$. These are $Q_{1}:=A_{1} B_{1} \cap A_{2} B_{2}$ and $Q_{2}:=A_{1} B_{2} \cap A_{2} B_{1}$. Since $|X|>1$, we have $X=\left\{Q_{1}, Q_{2}\right\}$. If $P$ were not collinear with $Q_{1}$ and $Q_{2}$, then $P Q_{i}$ would be a third tangent to $\mathcal{S}_{t}$ at $Q_{i}$, for $i=1,2$. It follows that the point set $\Pi_{2}:=\left\{P, A_{1}, A_{2}, B_{1}, B_{2}, Q_{1}, Q_{2}\right\}$ is a Fano subplane in $\Pi_{q}$.

To prove (c), define $Y \subseteq X$ as $Y:=\left\{A: A \in X,\left|A P \cap \mathcal{S}_{t}\right|=1\right\}$. The line set $\mathcal{L}$ contains $|Y|(t-1)$ tangents through the points of $Y$ and $(|X|-|Y|) t$ tangents through the points of $X \backslash Y$, hence

$$
|X|(t-1) \leqslant|X| t-|Y|=|\mathcal{L}|-\delta \leqslant t^{2},
$$

where $\delta$ denotes the number of non-tangent lines in $\mathcal{L}$. Because of (b), we may assume $t>2$, hence $|X| \leqslant t^{2} /(t-1)<t+2$ follows. To obtain a contradiction, suppose that $|X|=t+1$. If this is the case, then (9) implies $t \leqslant|Y|$. If $|Y|=t$, then $X \backslash Y$ consists of a unique point, but this contradicts the definition of $Y$. If $|Y|=t+1$, then $X=Y$ and through each point of $X$ there pass a non-tangent line, which is in $\mathcal{L}$. Thus if $\delta=1$, then the points of $X$ are contained in a line $\ell \in \mathcal{L}$. We may assume that $\ell=A_{t} B_{t}$. Then we can find $2(t-1)$ other non-tangent lines in $\mathcal{L}$, these are $A_{t} B_{i}$ and $B_{t} A_{i}$ for $i=1,2, \ldots, t-1$. On the other hand $\delta>1$ contradicts (9) and this contradiction proves $|X| \leqslant t$.

The following result shows some kind of stability of semiarcs containing a $V_{t}$-configuration.

Theorem 13. Let $\mathcal{S}_{t}$ be a $t$-semiarc in $\Pi_{q}, t<q$, and suppose that there exist two lines, $\ell_{1}$ and $\ell_{2}$, such that $\left|\ell_{1} \backslash\left(\mathcal{S}_{t} \cup \ell_{2}\right)\right|=n$ and $\left|\ell_{2} \backslash\left(\mathcal{S}_{t} \cup \ell_{1}\right)\right|=m$.

1. If $\ell_{1} \cap \ell_{2} \notin \mathcal{S}_{t}, t>1$ and $q>\min \{n, m\}+2 n m /(t-1)$, then $\mathcal{S}_{t}$ is of $V_{t}^{\circ}$ type.

2. If $\ell_{1} \cap \ell_{2} \in \mathcal{S}_{t}$ and $q>\min \{n, m\}+n m / t$, then $t=(q-1) / 2,\left|\mathcal{S}_{t}\right|=3(q+1) / 2$ and $\mathcal{S}_{t}$ is of $V_{t}^{\bullet}$ type. 
We have $n=m=t$ in both cases.

Proof. We may assume $m \geqslant n$. In part 1 , we have $n \geqslant t-1$, with equality only if $\ell_{2}$ is not a secant of $\mathcal{S}_{t}$, i.e. when $m \in\{q-1, q\}$. The assumption $q>\min \{n, m\}+2 n m /(t-1)$ implies $n, m<q-1$, hence this is not the case. It follows that $n, m \geqslant t$ holds. In part 2 , we have $n, m \geqslant t$, hence the assumption implies $n, m<q$ or, equivalently, the lines $\ell_{1}$ and $\ell_{2}$ are secants of $\mathcal{S}_{t}$. First we show $n=m=t$ in both cases. From this, part 1 follows immediately.

Suppose, contrary to our claim, that $m \geqslant t+1$. Denote by $P$ the intersection of $\ell_{1}$ and $\ell_{2}$. Let $\mathcal{N}=\left\{N_{1}, N_{2}, \ldots N_{q-n}\right\}$ be the set of points of $\left(\ell_{1} \backslash P\right) \cap \mathcal{S}_{t}$ and $\mathcal{M}=$ $\left\{M_{1}, M_{2}, \ldots M_{m}\right\}$ be the set of points of $\ell_{2} \backslash\left(\mathcal{S}_{t} \cup P\right)$. Let $X=\mathcal{S}_{t} \backslash\left(\ell_{1} \cup \ell_{2}\right)$. Through each point $N_{j} \in \mathcal{N}$ there pass exactly $m-t$ non-tangent lines that intersect $\ell_{2}$ in $\mathcal{M}$. Each of these lines contains at least one point of $X$. Denote the set of these points by $X\left(N_{j}\right)$. Then we have the following:

- $\left|X\left(N_{i}\right)\right| \geqslant m-t$, for $i=1,2, \ldots, q-n$,

- $X \supseteq \cup_{i=1}^{q-n} X\left(N_{i}\right)$,

- if $P \notin \mathcal{S}_{t}$, then each point of $X$ is contained in at most $m-t+1$ point sets of $\left\{X\left(N_{1}\right), \ldots, X\left(N_{q-n}\right)\right\}$,

- if $P \in \mathcal{S}_{t}$, then each point of $X$ is contained in at most $m-t$ point sets of $\left\{X\left(N_{1}\right), \ldots, X\left(N_{q-n}\right)\right\}$.

In part 1, we have the following lower bound for the size of $X$ :

$$
\frac{(q-n)(m-t)}{m-t+1} \leqslant|X| .
$$

On the other hand, through each point of $X$ there pass at least $t-1$ tangents that intersect both $\ell_{1} \backslash\left(\mathcal{S}_{t} \cup P\right)$ and $\mathcal{M}$. Hence we have:

$$
|X| \leqslant \frac{n m}{t-1}
$$

Summarizing these two inequalities we get:

$$
q \leqslant n+\frac{n m}{t-1}+\frac{n m}{(m-t)(t-1)} \leqslant n+\frac{2 n m}{t-1},
$$

that is a contradiction.

In part 2, observe that Lemma 10 and $q>\min \{m, n\}+n m / t$ together imply $n=t$. If $m \geqslant t+1$, then similarly to (10) and (11), we get $(q-t)(m-t) /(m-t) \leqslant|X|$ and $|X| \leqslant m t / t$ respectively. These two inequalities imply $q \leqslant t+m$, contradicting our assumption $q>\min \{n, m\}+n m / t=t+m$, hence $m=t$ follows. If $n=m=t$, then Lemma 10 implies $q \leqslant 2 t+1$ while our assumption yields $q>2 t$, thus $t=(q-1) / 2$. Since in this case there is equality in Lemma 10, we have $\left|\mathcal{S}_{t}\right|=3 q-3 t=3(q+1) / 2$. 
Let $\alpha_{n, m}$ and $\beta_{n, m}$ denote the lower bounds on $q$ in part 1 and in part 2 of Theorem 13 , respectively. The following example shows that the weaker assumptions $\alpha_{n, m}<3 q$ and $\beta_{n, m}<2 q$, respectively, do not imply the existence of a $V_{t}$-configuration contained in the semiarc.

Example 14. We give two examples for $t$-semiarcs, $\mathcal{S}_{t}$, such that they do not contain a $V_{t}$-configuration and there exist two lines, $\ell_{1}$ and $\ell_{2}$, with $\ell_{1} \backslash\left(\ell_{2} \cup \mathcal{S}_{t}\right)=t$ and $\ell_{1} \backslash\left(\ell_{2} \cup \mathcal{S}_{t}\right)=$ $t+1$. To do this, choose a conic $\mathcal{C}$ in $\Pi_{s}$, that is a projective plane of order $s>3$. Let $Q_{1}$ and $Q_{2}$ be two points of $\mathcal{C}$ and proceed as follows.

1. Let $\ell_{i}$ be the tangent of $\mathcal{C}$ at the point $Q_{i}$, for $i=1,2$, and denote $\ell_{1} \cap \ell_{2}$ by $P$. Take a point $Z \in Q_{1} Q_{2}$ such that $P Z$ is a secant of $\mathcal{C}$. Then $\mathcal{S}_{0}:=\left(\ell_{1} \cup \ell_{2} \cup \mathcal{C} \cup\{Z\}\right) \backslash\left\{P, Q_{2}\right\}$ is a point set without tangents. Now, if $\Pi_{s}$ is contained in $\Pi_{q}$, then $\mathcal{S}_{0} \subset \Pi_{s}$ is a $t$-semiarc in $\Pi_{q}$, with $t=q-s$. We have $\ell_{1} \cap \ell_{2} \notin \mathcal{S}_{t}$ and

$$
\alpha_{t, t+1}=(q-s)+2 \frac{(q-s+1)(q-s)}{q-s-1}<3 q .
$$

2. Let $\ell_{1}$ be the tangent of $\mathcal{C}$ at $Q_{1}$ and let $\ell_{2}$ be the line $Q_{1} Q_{2}$. Take a point $Z \in$ $\ell \backslash\left(\ell_{1} \cup \ell_{2}\right)$, where $\ell$ denotes the tangent of $\mathcal{C}$ at $Q_{2}$. Then $\mathcal{S}_{0}:=\left(\ell_{1} \cup \ell_{2} \cup \mathcal{C} \cup\{Z\}\right) \backslash\left\{Q_{2}\right\}$ is a point set without tangents. As before, if $\Pi_{s}$ is contained in $\Pi_{q}$, then $\mathcal{S}_{0} \subset \Pi_{s}$ is a $t$-semiarc in $\Pi_{q}$, with $t=q-s$. We have $\ell_{1} \cap \ell_{2} \in \mathcal{S}_{t}$ and

$$
\beta_{t, t+1}=(q-s)+\frac{(q-s+1)(q-s)}{q-s}<2 q .
$$

The next example is due to Suetake and it shows that when $t=1$, then there is no analogous result for part 1 of Theorem 13 .

Example 15 ([14], Example 3.3). Let $A$ be a proper, not empty subset of $\mathrm{GF}(q) \backslash\{0\}$, such that $A=-A:=\{-a: a \in A\}$ and $|A| \geqslant 2$. Let $B=\operatorname{GF}(q) \backslash(A \cup\{0\})$ and define the following set of points in $\operatorname{PG}(2, q)$ :

$$
\mathcal{S}_{1}:=\{(0, a, 1),(b, 0,1),(c, c, 1),(m, 1,0): a \in A, b \in B, c, m \in \mathrm{GF}(q) \backslash\{0\}, m \neq 1\} .
$$

Then $\mathcal{S}_{1}$ is a semioval with a $(q-1)$-secant, $X=Y$, and a $(q-2)$-secant, $Z=0$, intersecting each other not in $\mathcal{S}_{1}$. Also, $\mathcal{S}_{1}$ is not of $V_{1}^{\circ}$ type.

When $A=\mathrm{GF}(q) \backslash\{0\}$ in the above example, then $\mathcal{S}_{1}$ is a vertexless triangle with one point deleted from one of its sides. This example exists also in non-Desarguesian planes, but it is a semioval of $V_{1}^{\circ}$ type.

Semiarcs that properly contain a $V_{t}$-configuration exist in $\Pi_{q}$ whenever $\Pi_{q}$ contains a subplane. Some of the following examples were motivated by an example due to Korchmáros and Mazzocca (see [11], pg. 64). 
Example 16. Let $\Pi^{0}, \Pi^{1}, \ldots, \Pi^{s-1}$ be subplanes of $\Pi^{s}:=\Pi_{q}$ such that $\Pi^{i-1} \subset \Pi^{i}$ for $i=1, \ldots, s$. Denote by $r$ the order of $\Pi^{0}$ and let $\ell_{1}$ and $\ell_{2}$ be two lines in this plane. Let $P=\ell_{1} \cap \ell_{2}$ and set

$$
S(0):=\left(\ell_{1} \cup \ell_{2}\right) \cap\left(\Pi^{0} \backslash P\right), S(j):=\left(\ell_{1} \cup \ell_{2}\right) \cap\left(\Pi^{j} \backslash \Pi^{j-1}\right) \text {, for } j=1, \ldots, s .
$$

By $I$ we denote a subset of $\{1,2, \ldots, s\}$. We give four examples.

1. Let $\ell$ be a line in $\Pi^{0}$ passing through $P$ and let $Z$ be a subset of $\left(\ell \cap \Pi^{0}\right) \backslash\{P\}$ of size at least two. If $I$ is not empty, then $\mathcal{S}_{t}:=\cup_{j \in I} S(j) \cup Z$ is a $t$-semiarc of $V_{t}^{\circ}$ type with $t=q-\frac{1}{2} \sum_{j \in I}|S(j)|$.

2. Let $\ell$ be a line in $\Pi^{0}$ that does not pass through $P$ and let $Z$ be a subset of $\left(\ell \cap \Pi^{0}\right) \backslash\left(\ell_{1} \cup \ell_{2}\right)$ of size at least two. If $I$ is not empty, then $\mathcal{S}_{t}:=\cup_{j \in I} S(j) \cup Z$ is a $t$-semiarc of $V_{t}^{\circ}$ type with $t=q-\frac{1}{2} \sum_{j \in I}|S(j)|$.

3. Let $Z$ be a subset of $\Pi^{0} \backslash\left(\ell_{1} \cup \ell_{2}\right)$ such that there is no line in $\Pi^{0}$ passing through $P$ and meeting $Z$ in exactly one point. If $I$ is a proper subset of $\{1,2, \ldots, s\}$, then $\mathcal{S}_{t}:=\cup_{j \in I} S(j) \cup Z \cup S(0)$ is a $t$-semiarc of $V_{t}^{\circ}$ type with $t=q-r-\frac{1}{2} \sum_{j \in I}|S(j)|$.

4. Let $Z$ be a subset of $\Pi^{0} \backslash\left(\ell_{1} \cup \ell_{2}\right)$ such that for each line $\ell \neq \ell_{1}, \ell_{2}$ through $P$, $\ell$ is a line in $\Pi^{0}$, we have $|\ell \cap Z| \geqslant 1$. Then $\mathcal{S}_{t}:=\{P\} \cup S(0) \cup Z$ is a $t$-semiarc of $V_{t}^{\bullet}$ type with $t=q-r$.

\section{Semiarcs containing a $V_{t}$-configuration in $\mathrm{PG}(2, q)$}

In this section our aim is to characterize $t$-semiarcs containing a $V_{t}$-configuration in $\operatorname{PG}(2, q)$. We will need the following definition.

Definition 17. Let $\ell_{1}$ and $\ell_{2}$ be two lines in a projective plane and let $P$ denote their common point. We say that $X_{1} \subseteq \ell_{1} \backslash P$ and $X_{2} \subseteq \ell_{2} \backslash P$ are two perspective point sets if there is a point $Q$ such that each line through $Q$ intersects both $X_{1}$ and $X_{2}$ or intersects none of them. In other words, there is a perspectivity which maps $X_{1}$ onto $X_{2}$.

Lemma 18. Let $\mathcal{S}_{t}$ be a t-semiarc in $\Pi_{q}$ and suppose that $\left(\ell_{1} \triangle \ell_{2}\right) \cap \mathcal{S}_{t}$ is a $V_{t}$-configuration for some lines $\ell_{1}$ and $\ell_{2}$. If $\mathcal{S}_{t} \nsubseteq \ell_{1} \cup \ell_{2}$, then $\mathcal{S}_{t} \cap\left(\ell_{1} \backslash \ell_{2}\right)$ and $\mathcal{S}_{t} \cap\left(\ell_{2} \backslash \ell_{1}\right)$ are perspective point sets and each point of $\mathcal{S}_{t} \backslash\left(\ell_{1} \cup \ell_{2}\right)$ is the centre of a perspectivity which maps $\mathcal{S}_{t} \cap\left(\ell_{1} \backslash \ell_{2}\right)$ onto $\mathcal{S}_{t} \cap\left(\ell_{2} \backslash \ell_{1}\right)$.

Proof. Let $X=\mathcal{S}_{t} \backslash\left(\ell_{1} \cup \ell_{2}\right)$ and $X_{i}=\mathcal{S}_{t} \cap\left(\ell_{i} \backslash \ell_{j}\right)$, for $\{i, j\}=\{1,2\}$. For each $Q \in X$, if there were a line $\ell$ through $Q$ intersecting $X_{i}$ but not $X_{j}$, then the point $\ell \cap X_{i} \in \mathcal{S}_{t}$ would have at most $t-1$ tangents. This shows that each point of $X$ is the centre of a perspectivity which maps $X_{1}$ onto $X_{2}$. If $\mathcal{S}_{t} \not \ell_{1} \cup \ell_{2}$, then $X$ is not empty, hence $X_{1}$ and $X_{2}$ are perspective point sets. 
The following theorem characterizes perspective point sets in $\operatorname{PG}(2, q)$. This result was first published by Korchmáros and Mazzoca in [11] but we will use the notation of [4] by Bruen, Mazzocca and Polverino.

Theorem 19 ([4], Result 2.2, Result 2.3, Result 2.4, see also [11]). Let $\ell_{1}$ and $\ell_{2}$ be two lines in $\mathrm{PG}(2, q), q=p^{r}$, and let $P$ denote their common point. Let $X_{1} \subseteq \ell_{1} \backslash P$ and $X_{2} \subseteq \ell_{2} \backslash P$ be two perspective point sets. Denote by $U$ the set of all points which are centres of a perspectivity mapping $X_{1}$ onto $X_{2}$. Using a suitable projective frame in $\mathrm{PG}(2, q)$, there exist an additive subgroup $B$ of $\mathrm{GF}(q)$ and a multiplicative subgroup $A$ of $\mathrm{GF}(q)$ such that:

(a) $B$ is a subspace of $\mathrm{GF}(q)$ of dimension $h_{1}$ considered as a vectorspace over a subfield $\mathrm{GF}\left(q_{1}\right)$ of $\mathrm{GF}(q)$ with $q_{1}=p^{d}$ and $d \mid r$. This implies that $B$ is an additive subgroup of $\mathrm{GF}(q)$ of order $p^{h}$ with $h=d h_{1}$.

(b) $A$ is a multiplicative subgroup of $\mathrm{GF}\left(q_{1}\right)$ of order $n$, where $n \mid\left(p^{d}-1\right)$. In this way, $B$ is invariant under $A$, i.e. $B=A B:=\{a b: a \in A, b \in B\}$.

(c) If $G_{i}$ denotes the full group of affinities of $\ell_{i} \backslash P$ preserving the set $X_{i}, i=1,2$, then $G_{1} \cong G_{2} \cong G=G(A, B)=\{g: g(y)=a y+b, a \in A, b \in B\} \leqslant \Sigma$, where $\Sigma$ is the full affine group on the line $\mathrm{AG}(1, q)$.

(d) $X_{i}$ is a union of orbits of $G_{i}$ on $\ell_{i} \backslash P, i=1,2$, and $|U|=|G|=n p^{h}$.

(e) For every two integers $n, h$, such that $n \mid\left(p^{d}-1\right)$ and $d \mid \operatorname{gcd}(r, h)$, there exists in $\Sigma$ a subgroup of type $G=G(A, B)$ of order $n p^{h}$, where $A$ and $B$ are multiplicative and additive subgroups of $\mathrm{GF}(q)$ of order $n$ and $p^{h}$, respectively.

(f) $G$ has one orbit of length $p^{h}$ on $\mathrm{AG}(1, q)$, namely $B$, and $G$ acts regularly on the remaining orbits, say $\mathrm{O}_{1}, \mathrm{O}_{2}, \ldots, \mathrm{O}_{m}$, where

$$
m=\frac{q-p^{h}}{n p^{h}}=\frac{p^{r-h}-1}{n} .
$$

In the sequel we denote by $B^{i}$ the orbit of $G_{i}$ on $\ell_{i} \backslash P$ corresponding to $B$ and by $O_{1}^{i}, O_{2}^{i}, \ldots, O_{m}^{i}$ the remaining orbits, for $i=1,2$. With this notation $B^{1}$ is the image of $B^{2}$ under the perspectivities with centre in $U$ and also $O_{j}^{2}$ is the image of $O_{j}^{1}$ for $j=1,2, \ldots, m$ and vice versa.

(g) $B^{1} \subseteq X_{1}$ if and only if $B^{2} \subseteq X_{2}$ and the same holds for the other orbits, i.e. $O_{j}^{1} \subseteq X_{1}$ if and only if $O_{j}^{2} \subseteq X_{2}$, for $j=1,2, \ldots, m$.

(h) If a line $\ell$ not through $P$ meets $U$ in at least two points, then $\ell$ intersects both $B^{1}$ and $B^{2}$.

Exactly one of the following cases must occur. 
1. Both $A$ and $B$ are trivial. Then $U$ consists of a singleton.

2. $A$ is trivial and $B$ is not trivial. Then $U$ is a set of $p^{h}$ points all collinear with the point $P$.

3. $B$ is trivial and $A$ is not trivial. Then $U$ is a set of $n$ points on a line not through $P$.

4. $A$ and $B$ are the multiplicative and the additive group, respectively, of a subfield $\mathrm{GF}\left(p^{h}\right)$ of $\mathrm{GF}(q)$. Then

$$
U \cup B^{1} \cup B^{2} \cup\{P\}=\operatorname{PG}\left(2, p^{h}\right) .
$$

5. None of the previous cases occur. Then $U$ is a point set of size $n p^{h}$ and of type $\left(0,1, n, p^{h}\right)$, i.e. $0,1, n, p^{h}$ are the only intersection numbers of $U$ with respect to the lines in $\mathrm{PG}(2, q)$. In addition, using the fact that $|U|=n p^{h}$,

- there are exactly $n$ lines intersecting $U$ in exactly $p^{h}$ points and they are all concurrent at the common point $P$ of $\ell_{1}$ and $\ell_{2}$,

- each line intersecting $U$ in exactly $n$ points meets both $B^{1}$ and $B^{2}$.

Lemma 20 ([6], Proposition 3.1). If $\mathcal{S}_{t}$ is a $(q-2)$-semiarc in $\Pi_{q}$, then it is one of the following three configurations: four points in general position, the six vertices of a complete quadrilateral, or a Fano subplane.

In the next theorems we will use the notation of Theorem 19.

Theorem 21. Let $\mathcal{S}_{t}$ be a t-semiarc in $\mathrm{PG}(2, q), q=p^{r}$, and suppose that $\left(\ell_{1} \triangle \ell_{2}\right) \cap \mathcal{S}_{t}$ is a $V_{t}$-configuration for some lines $\ell_{1}$ and $\ell_{2}$. To avoid trivial cases, suppose that $\mathcal{S}_{t} \nsubseteq \ell_{1} \cup \ell_{2}$.

Let $X_{i}=\ell_{i} \cap \mathcal{S}_{t}$, for $i=1,2$, and let $X=\mathcal{S}_{t} \backslash\left(\ell_{1} \cup \ell_{2}\right)$. Also let $P=\ell_{1} \cap \ell_{2}$. Because of Lemma 18 we have that $X_{1}$ and $X_{2}$ are perspective point sets and $X \subseteq U$, where $U$ is the set of all points which are centres of a perspectivity mapping $X_{1}$ onto $X_{2}$. Choose a suitable coordinate system as in Theorem 19 and suppose that the size of $G=G(A, B)$ is $n p^{h}$, i.e. $|A|=n$ and $|B|=p^{h}$, where $A$ and $B$ are the multiplicative and the additive subgroup of $\mathrm{GF}(q)$ associated to the perspective point sets $X_{1}$ and $X_{2}$.

(I) If $P \notin \mathcal{S}_{t}$, i.e. $\mathcal{S}_{t}$ is of $V_{t}^{\circ}$ type, then one of the following holds.

(i) $X$ is contained in a line through $P$ that meets $U$ in $p^{h}$ points, $h \geqslant 1$, and we have $2 \leqslant|X| \leqslant p^{h}$,

(ii) $X$ is contained in a line not through $P$ that meets $U$ in $n \geqslant 2$ points and we have $2 \leqslant|X| \leqslant n$,

(iii) $|X| \geqslant 2$ and $X$ is a subset of $U$ such that there is no line through $P$ that meets $X$ in exactly one point. 
In the first two cases $X_{i}=\cup_{j \in I} O_{j}^{i}$ for some not empty subset $I \subseteq\{1,2, \ldots, m\}$ and for $i=1,2$. We have $t=q-k n p^{h}$, where $k=|I|$ and $1 \leqslant k \leqslant m$, where $m=\left(p^{r-h}-1\right) / n$.

In the third case $X_{i}=\cup_{j \in I} O_{j}^{i} \cup B^{i}$ for some proper subset $I \subset\{1,2, \ldots, m\}$ and for $i=1,2$. We have $t=q-k n p^{h}-p^{h}$, where $k=|I|, h \geqslant 1$ and $0 \leqslant k \leqslant m-1$.

(II) If $P \in \mathcal{S}_{t}$, i.e. $\mathcal{S}_{t}$ is of $V_{t}^{\bullet}$ type, then one of the following holds.

(i) $\mathcal{S}_{t}$ consists of the six vertices of a complete quadrilateral or $\mathcal{S}_{t}$ is a Fano subplane. We have $t=q-2$ in both cases.

(ii) $\ell_{1}$ and $\ell_{2}$ are lines in the subplane $\mathrm{PG}\left(2, p^{h}\right)$ and

$$
\mathcal{S}_{t}=\mathrm{PG}\left(2, p^{h}\right) \cap\left(\ell_{1} \cup \ell_{2}\right) \cup X,
$$

where $X$ is a subset of $\mathrm{PG}\left(2, p^{h}\right) \backslash\left(\ell_{1} \cup \ell_{2}\right)$ such that for each line $\ell \neq \ell_{1}, \ell_{2}$ through $P, \ell$ is a line in $\mathrm{PG}\left(2, p^{h}\right)$, we have $|\ell \cap X| \geqslant 1$. In this case $t=q-p^{h}$.

(iii) $\mathcal{S}_{t}$ is projectively equivalent to the following set of $3(n+1)$ points:

$$
\mathcal{S}_{t}:=\{(a, 0,1),(0,-a, 1),(a, 1,0): a \in A\} \cup\{(1,0,0),(0,1,0),(0,0,1)\},
$$

In this case $t=q-1-n$, where $n \mid q-1$.

The converse is also true, if $X_{1}$ and $X_{2}$ are perspective point sets and $X$ is as in one of the three cases in (I), then $X \cup X_{1} \cup X_{2}$ is a t-semiarc of $V_{t}^{\circ}$ type. If $\mathcal{S}_{t}$ is as in one of the three cases in (II), then $\mathcal{S}_{t}$ is a t-semiarc of $V_{t}^{\bullet}$ type.

Proof. We begin by proving (I). First assume $B^{1} \subseteq \ell_{1} \backslash X_{1}$. Then Theorem 19 (g) implies $B^{2} \subseteq \ell_{2} \backslash X_{2}$. Suppose that there exist three non-collinear points in $X$, say $L, M$ and $N$. Then between the lines $L M, L N$ and $M N$ there are at least two, say $L M$ and $L N$, not through $P$. Theorem 19 (h) and $X \subseteq U$ imply that these two lines intersect both $B^{1}$ and $B^{2}$. But then through $L$ there pass at most $t-1$ tangents, a contradiction. It follows that $X$ is contained in a line and hence it is as in one of our first two cases. The condition $|X| \geqslant 2$ comes from Proposition 12 (a).

Now asume $B^{1} \subseteq X_{1}$ and hence $B^{2} \subseteq X_{2}$. In this case for every two points $M, N \in X$, the line $M N$ intersects $\ell_{i}$ in $X_{i}$, for $i=1,2$. Thus the number of tangents through a point $L \in X$ is $t$ if and only if the line $L P$ contains at least one other point of $X$. Case 3 of Theorem 19 shows that this is not possible when $B$ is trivial, i.e. when $h=0$. Hence $X$ is as in our third case.

Now we prove (II). First assume $B^{1} \subseteq \ell_{1} \backslash X_{1}$ and hence $B^{2} \subseteq \ell_{2} \backslash X_{2}$. Suppose that there exist two points in $X$, say $M$ and $N$, not collinear with $P$. Then the line $M N$ intersects $\ell_{1}$ and $\ell_{2}$ not in $\mathcal{S}_{t}$. But then the number of tangents through $M$ is at most $t-1$, a contradiction. Thus $X$ is contained in a line through $P$ and through $P$ there pass exactly $q-2$ tangents. So $\mathcal{S}_{t}$ is a $(q-2)$-semiarc. According to Lemma $20, \mathcal{S}_{t}$ is as in (II)(i).

Now assume $B^{1} \subseteq X_{1}$ and hence $B^{2} \subseteq X_{2}$. In this case $t=q-k n p^{h}-p^{h}$ for some $k \in\{0,1, \ldots, m-1\}$, where $m$ is the number of orbits of $G$ of size $n p^{h}$ on $A G(1, q) \backslash B$. 
Since $P$ has exactly $t$ tangents, there are $q+1-t$ non-tangent lines through $P$. According to Theorem 19, we have $q-1-t \leqslant n$ and hence $k n p^{h}+p^{h}-1 \leqslant n$. We distinguish two subcases.

If $h>0$, then $n \mid p^{h}-1$ implies $n \leqslant p^{h}-1$ and hence $k n p^{h}=0$. This occurs only if $k=0$ and $n=p^{h}-1$. But $n$ divides $p^{d}-1$, where $d \mid h$ and $B$ is a subspace over the field $\operatorname{GF}\left(p^{d}\right)$. This implies $d=h$, thus $B$ is a subfield and $U$ is as in case 4 of Theorem 19 . This is only possible if $\mathcal{S}_{t}$ is as in our second case.

If $h=0$, then $k n \leqslant n$ and $U$ is as in case 3 of Theorem 19. If $k=0$, then $t=q-1$, which we excluded. Thus we have $k=1$ and $t=q-n-1$. This occurs only if $\mathcal{S}_{t}$ is as in our third case (see [4], pg. 56-57).

Theorem 22. Let $\mathcal{S}_{t}$ be a t-semiarc of $V_{t}^{\circ}$ type in $\mathrm{PG}(2, q), q=p^{r}$. Then the following hold.

(a) If $\operatorname{gcd}(q, t)=1$ and $\operatorname{gcd}(q-1, t-1)=1$, then $\mathcal{S}_{t}$ is a $V_{t}$-configuration.

(b) If $\operatorname{gcd}(q, t)=1$, then $\mathcal{S}_{t}$ is contained in a vertexless triangle.

(c) If $\operatorname{gcd}(q-1, t)=1$, then $\mathcal{S}_{t}$ is contained in a vertexless triangle or in the union of three concurrent lines without their common point.

Proof. We have $p^{h} \mid t$ in all three cases of Theorem 21 (I), where $p^{h}$ is the size of $B$. Hence $\operatorname{gcd}(q, t)=1$ implies $p^{h}=1$, i.e. $h=0$. This occurs only in the second case of Theorem 21 (I) and this proves (b).

In the first two cases of Theorem 21 (I) we have $n \mid(t-1)$ and hence also $n \mid \operatorname{gcd}(q-$ $1, t-1)$, where $n$ is the size of $A$. We have seen previously that $\operatorname{gcd}(q, t)=1$ can hold only in the second case of Theorem 21 (I). But in that case we have $n \geqslant 2$, which is a contradiction when $\operatorname{gcd}(q-1, t-1)=1$. This proves $(\mathrm{a})$.

If $\mathcal{S}_{t}$ is as in one of the first two cases of Theorem $21(\mathrm{I})$, then we are done. So to prove (c), it is enough to consider Theorem 21 (I)(iii). In this case $t=(q-1)-n k p^{h}-\left(p^{h}-1\right)$ and hence $n \mid \operatorname{gcd}(q-1, t)$. If $\operatorname{gcd}(q-1, t)=1$, then $n=1$, i.e. $A$ is trivial. If this happens, then case 2 of Theorem 19 implies that $\mathcal{S}_{t}$ is contained in the union of three concurrent lines without their common point.

\section{Acknowledgements}

The author is very grateful for the advices of Prof. Gábor Korchmáros and Prof. Tamás Szőnyi.

\section{References}

[1] D. Bartoli, G. Faina, Gy. Kiss, S. Marcugini, and F. Pambianco. 2-semiarcs in $\mathrm{PG}(2, q)$. Ars Combin., to appear.

[2] A. Blokhuis. On the size of a blocking set in PG(2,p). Combinatorica, 14(1):111-114, 1994. 
[3] A. Blokhuis, A. Brouwer, T. Szőnyi, and Zs. Weiner. On $q$-analogues and stability theorems. J. Geom., 101:31-50, 2011.

[4] A. A. Bruen, F. Mazzocca, and O. Polverino. Blocking Sets, Linear Groups and Transversal Designs. Quaderni di Matematica, 19:51-65, 2010.

[5] B. Csajbók, T. Héger, and Gy. Kiss. Semiarcs with a long secant in PG(2,q). Submitted. Available online at arXiv:1310.7207

[6] B. Csajbók and Gy. Kiss. Notes on semiarcs. Mediterr. J. Math., 9:677-692, 2012.

[7] J. M. Dover. Semiovals with large collinear subsets. J. Geom., 69:58-67, 2000.

[8] A. Gács, L. Lovász, and T. Szőnyi. Directions in $\mathrm{AG}\left(2, p^{2}\right)$. Innov. Incidence Geom., 6/7:189-201, 2009.

[9] J. W. P. Hirschfeld. Projective geometries over finite fields. Clarendon Press, Oxford, 1979, 2nd edition, 1998.

[10] Gy. Kiss. A survey on semiovals. Contrib. Discrete Math., 3(1):81-95, 2008.

[11] G. Korchmáros and F. Mazzocca. Nuclei of point sets of size $q+1$ contained in the union of two lines in $\mathrm{PG}(2, q)$. Combinatorica, 14(1):63-69, 1994.

[12] L. Lovász and A. Schrijver. Remarks on a theorem of Rédei. Studia Sci. Math. Hungar., 16:449-454, 1981.

[13] K. Metsch. Blocking sets in projective spaces and polar spaces. J. Geom., 76:216-232, 2003.

[14] C. Suetake. Some Blocking Semiovals which Admit a Homology Group. European J. Combin., 21:967-972, 2000.

[15] T. Szőnyi and Zs. Weiner. Proof of a conjecture of Metsch. J. Comb. Theory Ser. A, 118(7):2066-2070, 2011.

[16] Zs. Weiner. Geometric and algebraic methods in Galois-geometries. Ph.D. thesis, Eötvös University, Budapest, 2002. 